\title{
BioCAE: A New Strategy of Complex Biological Systems for Biofabrication of Tissues and Organs
}

\author{
Dernowsek JA' ${ }^{1,2 *}$, Rezende RA ${ }^{1-3}$ and JVL da Silva ${ }^{1,2}$
}

${ }^{1}$ Center for Information Technology Renato Archer (CTI), 3D Technologies Research Group (NT3D) Rodovia D. Pedro I (SP - 65) Km 143,6, Amarais, Campinas, SP, 13.069-901, Brazil

${ }^{2}$ Brazilian Institute of Biofabrication (INCT-BIOFABRIS), School of Chemical Engineering, UNICAMP, Campinas, SP, Brazil

${ }^{3}$ CERTBIO, Federal University of Campina Grande (UFCG), Campina Grande, PB, Brazil

\begin{abstract}
Biofabrication as an interdisciplinary area is fostering new knowledge and integration of areas like nanotechnology, chemistry, biology, physics, materials science, control systems, among many others, necessary to accomplish the challenge of bioengineering functional complex tissues. The emergence of integrated platforms and systems biology to understand complex biological systems in multiscale levels will enable the prediction and creation of biofabricated biological structures. This systematic analysis (meta-analysis) or integrated platforms for estimating biological process have been named as BioCAE, which will become the key for important steps of the biofabrication processes. Biological Computational Aided Engineering (BioCAE) is a new computational approach to understanding and bioengineer complex tissues (biofabrication) using a combination of different methods as multiscale modelling, computer simulations, data mining and systems biology. In addition, multi-agent systems (MAS), which are composed of different interacting computing entities called agents, also provide an interesting way to design and implement simulations of biological systems, integrating them with all steps of the BioCAE. MAS as a part of computational science have become a growing area to manipulate and solve complex problems. This paper presents an approach that will allow predicting the development and behavior of different biological processes such as molecular networks, gene interactions, cells, tissues and organs due to its flexibility, beyond to provide a new outlook in the biofabrication of tissues and organs.
\end{abstract}

Keywords: Biological computational aided engineering (BioCAE); Biofabrication; Information technology; Systems biology; Computer simulations; Multi-agent system

\section{Introduction}

Biofabrication as an interdisciplinary area is fostering new knowledge and integration of areas like nanotechnology, chemistry, biology, physics, materials science, control systems, among many others, necessary to accomplish the challenge of bioengineering functional complex tissues. The term biofabrication was widely discussed recently and is broadly used for tissue engineering and additive manufacturing as "the automated generation of biologically functional products with structural organization from living cells, bioactive molecules, biomaterials, cell aggregates such as micro-tissues or hybrid cell-material constructs, through Bioprinting or Bioassembly and subsequent tissue maturation processes" [1]. Undoubtedly, the current biofabrication initiatives are possible because of a set of technologies that are completely supported by information technology (IT), such as imaging, database, bioprinting technique [2], artificial intelligence (AI), software and hardware. These factors make the biofabrication not only a consistent promise but also a reality for the automated production of human tissues and organs.

The bioengineering of functional tissues, functional vascular networks, interfaces, structural hierarchy and complex features is emerging as an unparalleled scientific and technical challenge for the next generation of tissue engineers [3]. However, advances in molecular and cellular biology, over the last decades, have triggered tremendous growth in the availability of experimental data. On the other hand, the computational approaches have allowed the multiparametric analyses of various datasets and biological behaviors. This is, therefore, the first engine to leverage the main goal of bioengineering tissues and organs. It begins with a deep knowledge of the behavior of the cell, mainly their mechanisms to connect molecules and machinery, aiming at activating, sustaining and modulating their functional metabolic networks responsible for the survival, growth, proliferation, differentiation and death [4].

The large-scale high-throughput experimental techniques have greatly increased overall knowledge and understanding of the global organization of complex cross-talks among the different signaling cascades and different cells. Recognizing this complexity, the concept of complex biological systems or systems biology has emerged as a powerful field that requires the ability to mathematics, engineering and information technology domains to analyze, integrate and use data from different databanks for the creation of working models of entire biological systems [5]. The ultimate outcome of this structural and functional organization is the metabolism of the cells, tissues and organs, important in all biofabrication processes. This technology is evolved into a complex system composed of many processes as computer-aided design (CAD), computer aided engineering (CAE) and computer aided manufacturing $(\mathrm{CAM})$ and biological processes, which depends on the combination of different interrelated components as molecules, genes, regulatory networks, cells, organoids and tissues, integrated with computational approaches as design, modeling, simulation and optimization, among others.

Next sessions we expose a new approach, based on Information

*Corresponding author: Janaina de Andrea Dernowsek, Center for information technology Renato Archer (CTI), 3D Technologies Research Group (NT3D), Campinas, Brazil, Tel: +55-19-3746-6203; E-mail: janaina.dernowsek@cti.gov.br

Received April 29, 2017; Accepted June 07, 2017; Published June 17, 2017

Citation: Dernowsek JA, Rezende RA, da Silva JVL (2017) BioCAE: A New Strategy of Complex Biological Systems for Biofabrication of Tissues and Organs. J Tissue Sci Eng 8: 200. doi: 10.4172/2157-7552.1000200

Copyright: @ 2017 Dernowsek JA, et al. This is an open-access article distributed under the terms of the Creative Commons Attribution License, which permits unrestricted use, distribution, and reproduction in any medium, provided the original author and source are credited. 
Technology that potentially will help to improve understanding, integration and consequently optimizing the output of the biofabrication process.

\section{Integrated Platform for Biofabrication}

The emergence of platforms and systems biology to understand biological systems in multiscale levels will enable the prediction and the creation of biofabricated biological structures [6]. This systematic analysis (meta-analysis) or integrated platforms for predicting the biological behavior has been named BioCAE (Figure 1) and together with BioCAD and BioCAM will become the key procedures for establishing essential steps of the biofabrication processes.

Biological Computational Aided Engineering (BioCAE) is a new computational approach to understanding and bioengineer complex tissues (biofabrication) using a combination of different methods as multi-scale modeling, computer simulations, data mining and systems biology (Figure 1). In addition, multi-agent systems (MAS), which are composed of different interacting computing entities called agents, also provide an interesting way to design and implement simulations of biological systems [7], integrating them with all steps of the BioCAE. MAS, as a part of computational science, has become a growing area for the manipulation and solving complex problems [8]. This paper, presents a new integrated approach to predict the development and behavior of different biological processes such as molecular networks, intracellular interactions (including genes, mRNAs, microRNAs and proteins), cell-cell interactions and tissues and organs, besides to provide a new landscape in the design of functional organs and tissues for the next step of their production by means of bioprinting processes.

\section{Biological Computational Aided Engineering for Biofabrication (BioCAE)}

BioCAE will be an integrated environment where several modules of modeling and simulation are interoperated to understand and predict a suitable and functional organ design (BioCAD) for biofabrication (BioCAM). In other words, today, great efforts and high costs are required to, experimentally, manipulate cells and/or cell agglomerates that will especially necessary for the biofabrication of organs and tissues. Therefore, the use and the development of computational approaches to study complex biological systems in different levels (cells, tissues and organs) in silico are fundamental for the progress of the unique biofabrication field.

The emergence of powerful software platforms to study the biological behaviors in different environments, as BioCAE, depends on data handling/data mining, imaging, computer modeling, computer simulation, integrated computational analysis, MAS and knowledge integration, among many others. One of the main approaches of our proposal is to create computational models that enable users to predict the behaviors of biological systems at multi-scales, thereby helping users to understand the mechanisms involved as well as to predict the impacts that occur during the biofabrication process. It includes a myriad of parameters such as pressures, $\mathrm{pH}$, oxygenation, nutrition, fusion, diffusion, temperature, stresses, velocity and viscosity, among many others that affect biological viability in the survival, growth, proliferation and differentiation.

Many papers highlight the importance of computational multi-scale methods in systems biology [9-13] and recently, in the biofabrication [14]. The biological systems are made up of many spatial and temporal scales. These diverse levels coupling intra and interscale interactions make a biological system extremely complex, requiring advanced mathematical and computational models for the integration of the different scales $[15,16]$. There is a range of multi-scale modelling methods that could potentially be employed in systems biology [17-22] and consequently in the biofabrication of tissues and organs.

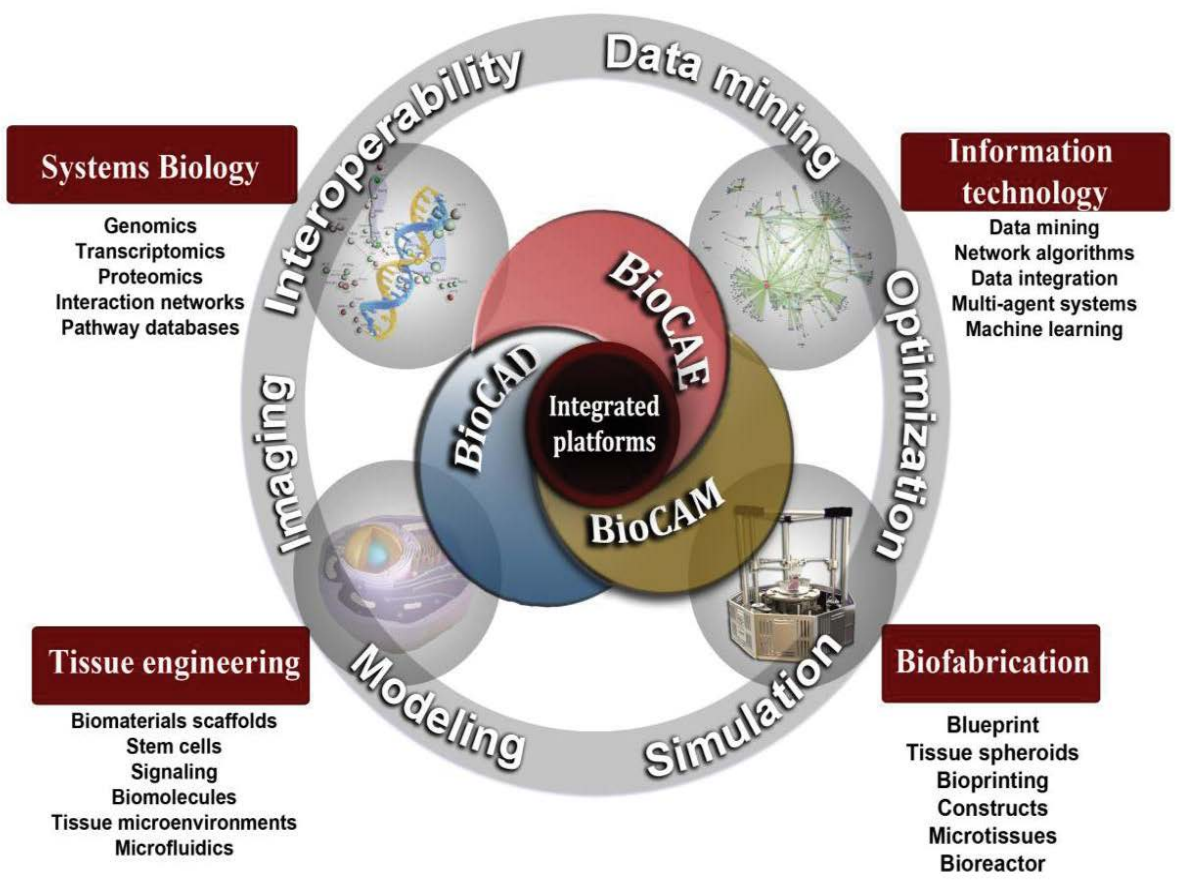

Figure 1: Illustration of how BioCAD, BioCAE and BioCAM concepts, together with tissue engineering principles, systems biology and information technology (IT) can synergically benefit to establish the core for the biofabrication. 
Then, in the next section, we will describe some multi-scale approaches and/or software which may be part of the BioCAE and that are playing or will play important roles in multi-scale problems in systems biology and tissues biofabricated, thereby preventing a large amount of trial and error experiments in laboratories.

\section{From Molecules, Cells and Tissues towards Organ}

The aim of multi-scale modelling is to represent, predict and understand the behaviour of complex systems across, not only in a wide spatial scale (molecules, genes, mRNAs, proteins, metabolic networks, cells, organoids, tissues and organs) as shown in Figure 2, but also in temporal scale (seconds (s), minutes ( $\mathrm{min}$ ), hours (h), days, weeks, months, years). Despite the significant growth of this field of knowledge, there is a need to develop frameworks for the interoperability among different simulation software. In this context, it is possible to find that several toolkits, databases, pipelines, methods, integrated frameworks and platforms have been developed for this purpose [23]. On the other hand, the major drawback of these approaches is the high computational requirements, particularly for higher eukaryotes, where even a single organ is composed of a large number of cells. Many approaches share mathematical models, especially when using integrated platforms or software are used. Accordingly, various methods are presented below, which were developed for the study of complex biological systems that can be adapted to compose the BioCAE concept.
For a better understanding, the following three sections describe a multilevel approach, from molecules to organs that will be integrated with relevant information and models using the BioCAE framework proposal.

\section{Cell Modelling Level: From Molecules to Cells}

Because of the intrinsic nature of biological systems and their processes (sequences, genes, proteins, signaling networks, regulatory networks and metabolic networks), the unequivocal identification of a molecule or sequence in a specific process is nontrivial. In contrast, the vast amount of experimental data and high throughput analyses (genomics, transcriptomics, miRNomics and proteomics) has identified a myriad of key factors (genes, RNAs, microRNAs, proteins and pathways). With this in mind, tissue bioengineers and other researchers who wish to biofabricate tissues and organs should understand firstly how molecular-scale mechanisms lead to complex functional structures at the scale of tissues, organs and organisms.

The characterization of intracellular pathways and their behaviour are the original focus of systems biology and it has a long history of work and achievement in the development and in the use of mathematical models of cellular signalling, metabolic control and MAS to set up simulations for molecular and cellular biology [10]. Equally important are the graphical diagrams of biological processes, which are useful for visual presentation, mainly for biologists. In terms of

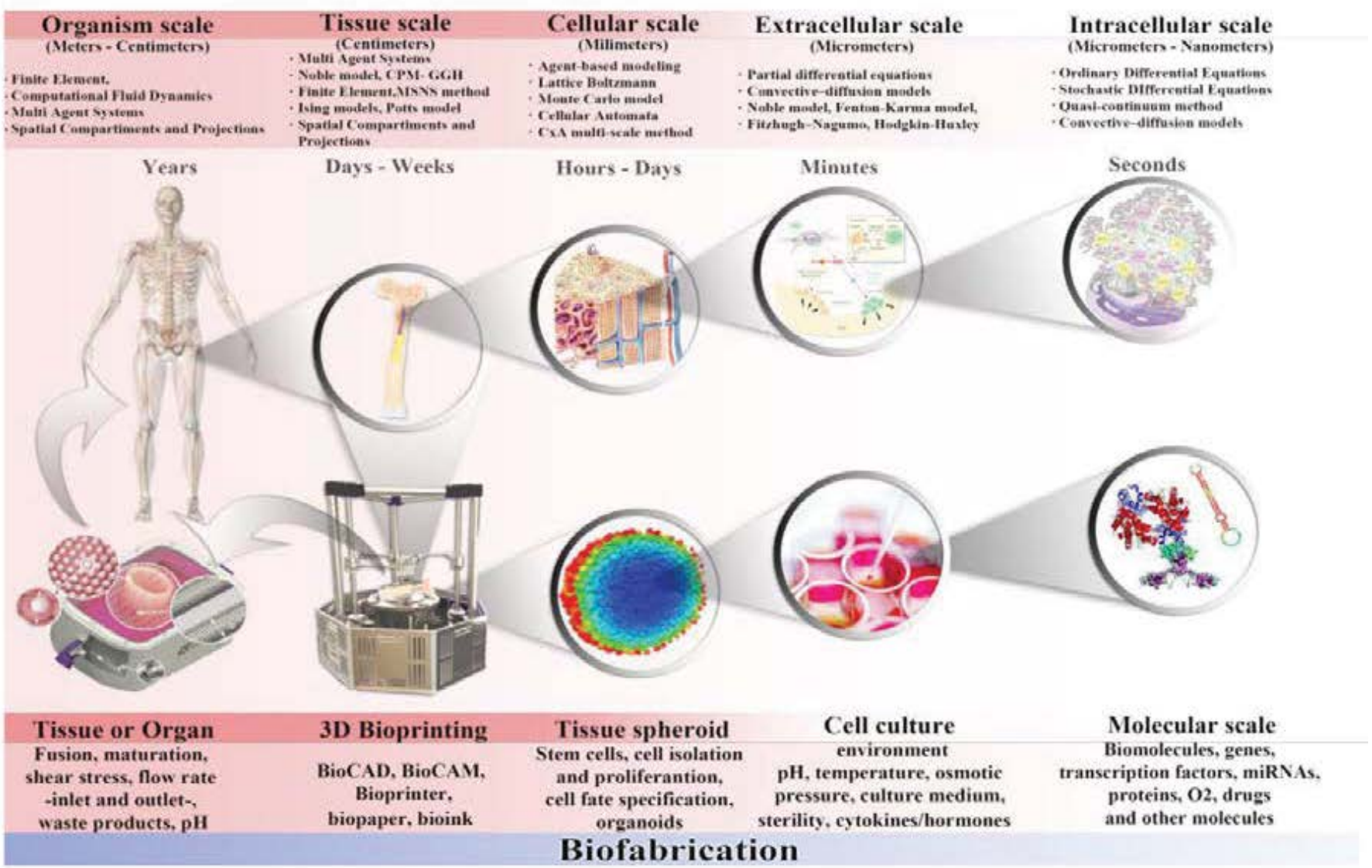

Figure 2: Computational and mathematical methods to study complex models at several levels of biological organization - BioCAE - (molecules, genes, mRNAs, proteins, metabolic networks, cells, organoids, tissues, and organs), which can be adapted to predict the development of a tissue and/or organ in many steps of the biofabrication. 
software, a different representation format is needed for quantifying a model for simulation and analysis. This representation can use a standard language called Systems Biology Markup Language (SBML) [24]. SBML is a machine-readable format for representing models and it is suitable for representing models commonly found in research on a number of topics, including cell signalling pathways, metabolic pathways, biochemical reactions, gene regulation and many others [25].

Another possibility to comprehend these phenomena would be by means of specialized frameworks, for example, the CompuCell3D (CC3D), which is multi-model software that simulates interactions of a gene regulatory network with cellular mechanisms, cell adhesion, haptotaxis and chemotaxis, among others. The three main background components of CC3D are a Cellular Potts Model (CPM), a ReactionDiffusion (RD) module and a combined ODE/state model of genetic regulatory networks and differentiation [26].

The crucial step to the computational modeling is to cast the model into a standardized, computable format that can be analyzed rigorously using simulations and mathematical methods, whereas the different representations of models are useful for different purposes. For this reason, many research groups have developed approaches for studying biological processes related to the intracellular and extracellular environment, as secretion, absorption, diffusion of chemicals with the environment, cell proliferation, cell differentiation, adhesion, cell movement and cell internal dynamics. Below are described some of these models found in the literature:

i) Chemical diffusion: the process of molecule secretion and absorption as facilitated diffusion. The literature lacks information about the transport mechanisms of such molecules as genes, transcription factors microRNAs, proteins and about the rate of diffusion. However, the following approaches can be used to study these processes: Quasi-continuum (GC), Quantum Mechanics-Molecular Mechanics (QM-MM), Computational Fluid Dynamic (CFD), Equation-free Multiscale (EFM), Kinetic Monte Carlo (KMC), Lattice Boltzmann (LB) and Molecular Dynamics Codes (MDC).

ii) Regulatory network: the replication, transcription, translation from the perspective of DNA and RNA begin with the binding at the gene promoter of one or more transcription factors. However, these intrinsic regulations might also be repressed by transcription factors and microRNAs [27]. This activation/inhibition is stochastic and highly depends on the concentration of molecules and sequences. Therefore, the probability of molecular mechanisms is a sum of positive and negative contributions from the concentration of enhancers and silencers, respectively [28]. Logical models (Boolean models, Probabilistic models and Bayesian Models) and Continuous Models (Linear Model and Differential equation based model) are the mathematical and statistical methods most commonly found in the regulatory networks prediction platforms [29].

iii) Migration and adhesion: The model of cell movement and adhesion considers the chemotaxis phenomenon, which is known to be responsible for cell sorting during morphogenesis and this model component is inspired by a previous work that considers chemotaxis as an important actor for the creation of self-organized structures $[30,31]$. The latest models proposed in the literature to describe different events in cellular adhesion and migration are based on Cellular Automata (CA) and Cellular Potts Models [32,33]. iv) Mitosis: cell division is fast and synchronous until cleavage, then slows down and becomes asynchronous. The rate of division is constant in the first hours of cell development and then decreases until a low value [34]. Mogilner et al. showed some related models with mitosis, among them, stochastic and transient microtubes - motor assemblies to drive steady, accurate movements during the process [35].

\section{Tissue Modelling Level: From Cells to Tissues}

Tissue-scale models explain how local interactions within and between cells lead to complex biological patterning where some methods have already been mentioned before. However, the two main approaches to tissue modeling are continuum models, which use celldensity fields and partial differential equations (PDEs) to model cell interactions without explicit representations of cells and MAS, which represent individual cells and interactions explicitly [17]. Furthermore, the most promising family of mathematical models in tissue simulation is those based on the Ising model, the Potts model and the CPMGGH model. The frameworks TSIM (http://www.tsimsoftware.com), Morpheus (https://imc.zih.tu-dresden.de/wiki/morpheus/doku. php?id=start), Simmune modeler (https://www.niaid.nih.gov/research/ simmune-project), CellSys (http://ms.izbi.uni-leipzig.de/index.php/ software/cellsys2010) and CC3D (http://www.compucell3d.org/) are some solutions that use this family of models.

\section{Organs Modelling Level: From Tissues to Organs}

A biological organ is a group of tissues joined in structure unit to perform a specific function or functions (e.g. heart, brain, kidney, lungs, etc.). Various processes at the tissue, cellular, sub-cellular and

\begin{tabular}{|l|c|c|}
\hline Model & Strategy & References \\
\hline $\begin{array}{l}\text { Modelling and simulation of diffusion process in tissue } \\
\text { spheroids }\end{array}$ & CFD & {$[14]$} \\
\hline Agent-based virtual tissue simulations & CC3D & {$[17]$} \\
\hline $\begin{array}{l}\text { Cell compressibility, motility and contact inhibition on the } \\
\text { growth of tumor cell clusters }\end{array}$ & CC3D & {$[18]$} \\
\hline $\begin{array}{l}\text { Multiscale modeling of the early CD8 T cell immune } \\
\text { response in lymph nodes }\end{array}$ & CC3D & {$[19]$} \\
\hline Multi-scale knowledge on cardiac development & CC3D & {$[20]$} \\
\hline The cell behavior ontology & CC3D & {$[21]$} \\
\hline Cell differentiation in the transition to multicellularity & CC3D & {$[22]$} \\
\hline Dynamics of cell aggregates fusion & CC3D & {$[36]$} \\
\hline A multi-cell model of tumor evolution & CC3D & {$[37]$} \\
\hline Virtual tissues & MAS & {$[38]$} \\
\hline Disruption of blood vessel development & CC3D & {$[39]$} \\
\hline Tumor growth and angiogenesis & CC3D & {$[40]$} \\
\hline Agent-oriented in silico liver (ILS) & MAS & {$[41]$} \\
\hline Model of thrombus development & MAS (TS) & {$[42]$} \\
\hline Three-dimensional multi-scale tumor model & MAS & {$[43]$} \\
\hline $\begin{array}{l}\text { A multi-scale model of dendritic cell education and } \\
\text { trafficking in the lung }\end{array}$ & CM & {$[44]$} \\
\hline Multi-scale model of follicular development & CM & {$[45]$} \\
\hline Multi-scale in silico leukocytes model & MAS & {$[46]$} \\
\hline Multi-scale model of organogenesis & CM & {$[47]$} \\
\hline Limitations of spheroids under inappropriate conditions & FE & {$[48]$} \\
\hline $\begin{array}{l}\text { Finite element modelling of the viscoelastic human } \\
\text { cranial cavity }\end{array}$ & FE & {$[49]$} \\
\hline CM: Continuum Mode; SH: Spatilly Hierarcical; TS: & Femporaly Separed; \\
\hline
\end{tabular}

CM: Continuum Model; SH: Spatially Hierarchical; TS: Temporally Separated; CC3D: CompuCell 3D; CP: Cellular Potts; FE: Finite element; CFD: Computational Fluid Dynamics; MAS: Multi-Agent System

Table 1: Summary of models of the biological system and the strategies used. 
lower levels occur at multiple time scales influencing the behavior of the organ. Multi-scale models are usually based on the continuum modeling and/or MAS approaches which can be decomposed into $\mathrm{N}$ single-scale mathematical models and several physical processes. Various works have already been done which apply many multi-scale methods in several biological systems [14,17-22,36-49]: a good review is proposed in Table 1 .

\section{Conclusion}

Systems biology aims at describing and understands the complex biological systems and ultimately to develop predictive models of human disease, meanwhile, our long-term goal is to develop a new strategy using the information technology to model, simulate and predict behaviours of a biological system for biofabrication of tissues and organs. Computational models can be used to explicitly represent a set of entities with a complex internal behaviour, which interacts with the others and with the environment generating an emergent behaviour representing the system dynamics; under those circumstances, many fields of knowledge will obtain essentials biological insights.

The BioCAEapproachintends to integratethevarious modelinglevels by means of relevant information among them. This accomplishment requires integration of knowledge from diverse biological components, data and methods that span several spatial and temporal scales into models of the system as a whole, though an important difficulty in multi-scale modeling is their high computational costs and integration. Therefore, the development of functional tissues and organs will only be possible with the emergence of a BioCAE framework that will take advantage of the huge amount of available models.

\section{Acknowledgement}

Our sincere thanks to the National Council for Scientific and Technological Development (CNPq) and FAPESP for the Brazilian Institute of Biofabrication (INCT-BIOFABRIS process 2008/57860-3) for financial support. The authors are also thankful to CNPq for the "Regenerative Medicine" grant (process 467643/2014 8). The authors are thankful to FAPESP for the Brazilian Research Institute for Neuroscience and Neurotechnology - BRAINN (CEPID process 2013/07559-3), for the Thematic Project (Grant 2011/22749-8). The authors are grateful to the whole team for the cooperation in the creation of images and table which are original and used for the first time.

\section{References}

1. Groll J, Boland T, Blunk T, Burdick JA, Cho DW, et al. (2016) Biofabrication Reappraising the definition of an evolving field. Biofabrication 8: 013001

2. Rezende RA, Kasyanov V, Mironov V, Silva JVL (2015) Organ printing as an information technology. Procedia Eng 110: 151-158.

3. Mikos AG, Herring SW, Ochareon P, Elisseeff J, Lu HH, et al. (2006) Engineering complex tissues. Tissue Engineering 12: 3307-3339.

4. Pieroni E, de la Fuente van Bentem S, Mancosu G, Capobianco E, Hirt H et al. (2008) Protein networking: insights into global functional organization of proteomes. Proteomics 8: 799-816.

5. Spivey A (2004) Systems biology: The big picture. Environ Health Perspect 112: A938-943.

6. Dernowsek JA., Rezende RA, Silva JVL (2017) The role of information technology (IT) in the future of 3D biofabrication. Journal of 3D Printing in Medicine 1: 63-74.

7. Wooldridge M, Wooldridge MJ (2002) An introduction to multiagent systems. John Wiley and Sons, p: 314

8. Chakraborty S, Gupta S (2014) Medical application using multi-agent system - a literature survey. International Journal of Engineering Research and Applications 4: 528-546.

9. Schnell S, Maini PK, Newman SA, Newman TJ (2008) Multiscale modeling of developmental systems. Introduction. Curr Top Dev Biol 81: xvii-xxv.
10. An G, Mi Q, Dutta-Moscato J, Vodovotz Y (2009) Agent-based models in translational systems biology. Wiley Interdiscip Rev Syst Biol Med 1: 159-171.

11. Dada JO, Mendes $P$ (2011) Multi-scale modelling and simulation in systems biology. Integr Biol (Camb) 3: 86-96.

12. Sbalzarini IF (2013) Modeling and simulation of biological systems from image data. Bioessays 35: 482-490.

13. Murfee WL, Sweat RS, Tsubota K, Gabhann FM, Khismatullin D, et al. (2015) Applications of computational models to better understand microvascular remodeling: A focus on biomechanical integration across scales. Interface Focus 5: 20140077.

14. Dernowsek JA, Rezende RA, Passamai VE, Noritomi PY, Kemmoku DT, et al. (2016) Modeling and simulation of diffusion process in tissue spheroids encaged into microscaffolds (lockyballs). Proceedings of 26th European Symposium on Computer Aided Process Engineering - ESCAPE. Slovenia, June 2016. Computer Aided Chemical Engineering 38: 1737-1742.

15. An G, Faeder J, Vodovotz Y (2008) Translational systems biology: introduction of an engineering approach to the pathophysiology of the burn patient. J Burn Care Res 29: 277-285.

16. Vodovotz Y, Csete M, Bartels J, Chang S, An G (2008) Translational systems biology of inflammation. PLoS Comput Biol 4: e1000014.

17. Swat M, Glazier JA, (2013) Agent-based virtual tissue simulations. Biomedical Computation Review Fall, pp: 28-29.

18. Li JF, Lowengrub J (2014) The effects of cell compressibility, motility and contact inhibition on the growth of tumor cell clusters using the Cellular Potts Model. J Theor Biol 343: 79-91.

19. Prokopiou SA, Barbarroux L, Bernard S, Mafille J, Leverrier Y, et al. (2014) Multiscale modeling of the early CD8 $\mathrm{t}$-cell immune response in lymph nodes: An Integrative study. Computation 2:159-181.

20. De Boer BA, Le Garrec JF, Christoffels VM, Meilhac SM, Ruijter JM (2014) Integrating multi-scale knowledge on cardiac development into a computational model of ventricular trabeculation. Wiley Interdiscip Rev Syst Biol Med 6: 389 397.

21. Sluka JP, Shirinifard A, Swat M, Cosmanescu A, Heiland RW, et al. (2014) The cell behavior ontology: Desczribing the intrinsic biological behaviors of real and model cells seen as active agents. Bioinformatics 30: 2367-2374.

22. Mora Van Cauwelaert E, Arias Del Angel JA, Benítez M, Azpeitia EM (2015) Development of cell differentiation in the transition to multicellularity: $A$ dynamical modeling approach. Front Microbiol 6: 603.

23. Turene N (2011) Role of a Web-based software platform for systems biology. $J$ Comput Sci Syst Biol 4: 35-41.

24. Hucka M, Finney A, Sauro HM, Bolouri H, Doyle JC, et al. (2003) The systems biology markup language (SBML): A medium for representation and exchange of biochemical network models. Bioinformatics 19: 524-531.

25. Hucka M, Finney A, Bornstein BJ, Keating SM, Shapiro BE, et al. (2004 ) Evolving a lingua franca and associated software infrastructure for computational systems biology: The Systems Biology Markup Language (SBML) project. Systems Biology 1: 41-53.

26. Izaguirre JA, Chaturvedi R, Huang C, Cickovski T, Coffland J, et al. (2004) CompuCell, a multi-model framework for simulation of morphogenesis. Bioinformatics 20: 1129-1137.

27. Dernowsek JA, Pereira MC, Fornari TA, Macedo C, Assis AF, et al. (2017) Posttranscriptional interaction between miR-450a-5p and miR-28-5p and STAT1 mRNA triggers osteoblastic differentiation of human mesenchymal stem cells. $\mathrm{J}$ Cell Biochem 1-18.

28. Robinson A, Van Oijen AM (2013) Bacterial replication, transcription and translation: Mechanistic insights from single-molecule biochemical studies. Nat Rev Microbiol 11: 303-315.

29. Vijesh N, Chakrabarti S, Sreekumar J (2013) Modeling of gene regulatory networks: A review. J Biomed Sci Eng 6: 223-231.

30. Davies J (2005) Mechanisms of morphogenesis. Academic Press Oxford, p: 414

31. Eyiyurekli M, Bai L, Lelkes PI, Breen D (2010) Chemotaxis-based sorting of self-organizing heterotypic agents. In: Proceeding of ACM Symposium on Applied Computing, pp: 1315-1322. 
Citation: Dernowsek JA, Rezende RA, da Silva JVL (2017) BioCAE: A New Strategy of Complex Biological Systems for Biofabrication of Tissues and Organs. J Tissue Sci Eng 8: 200. doi: 10.4172/2157-7552.1000200

Page 6 of 6

32. Vivas J, Garzón-Alvarado D, Cerrolaza M. (2015) Modeling cell adhesion and proliferation: A cellular-automata based approach. Adv Model Simul Eng Sci 2: 32.

33. Scianna M, Preziosi L, Wolf K (2013) A Cellular Potts Model simulating cell migration on and in matrix environments. Math Biosci Eng 10: 235-261.

34. Montagna S, Omicini A, Ricci A (2010) A multiscale agent-based model of morphogenesis in biological systems. Workshop on nazionale "Dagli Oggetti agli Agenti" WOA, Rimini, Italy.

35. Mogilner A1, Wollman R, Civelekoglu-Scholey G, Scholey J (2006) Modeling mitosis. Trends Cell Biol 16: 88-96.

36. Thomas GL, Mironov V, Nagy-Mehez A, Mombach JCM (2014) Dynamics of cell aggregates fusion: Experiments and simulations. Physica A 395: 247-254.

37. Swat MH, Thomas GL, Shirinifard A, Clendenon SG, Glazier JA (2015) Emergent stratification in solid tumors selects for reduced cohesion of tumor cells: A multi-cell, virtual-tissue model of tumor evolution using compucell 3D. PLoS ONE 10: e0127972.

38. Shah I, Wambaugh J (2010) Virtual tissues in toxicology. J Toxicol Environ Health B Crit Rev 13: 314-328.

39. Kleinstreuer N, Dix D, Rountree M, Baker N, Sipes N, et al. (2013) A computational model predicting disruption of blood vessel development. PLoS Comput Biol 9: e1002996.

40. Shirinifard A, Gens JS, Zaitlen BL, PopÅ,awski NJ, Swat M, et al. (2009) 3D multi-cell simulation of tumor growth and angiogenesis. PLoS ONE 4: e7190.

41. Yan L, Ropella GE, Park S, Roberts MS, Hunt CA (2008) Modeling and simulation of hepatic drug disposition using a physiologically based, multiagent in silico liver. Pharm Res 25: 1023-1036.

42. Xu Z, Chen N, Kamocka MM, Rosen ED, Alber M (2008) A multiscale model of thrombus development. J R Soc Interface 5: 705-722.

43. Zhang L, Athale CA, Deisboeck TS (2007) Development of a three-dimensiona multiscale agent-based tumor model: Simulating gene-protein interaction profiles, cell phenotypes and multicellular patterns in brain cancer. J Theor Bio 244: 96-107.

44. Klinke DJ 2nd1 (2007) A multi-scale model of dendritic cell education and trafficking in the lung: Implications for T cell polarization. Ann Biomed Eng 35: 937-955.

45. Echenim N, Monniaux D, Sorine M, Clément F (2005) Multi-scale modeling of the follicle selection process in the ovary. Math Biosci 198: 57-79.

46. Tang J, Ley KF, Hunt CA (2007) Dynamics of in silico leukocyte rolling, activation and adhesion. BMC Syst Biol 1: 14.

47. Cox B (2010) A multi-scale, discrete-cell simulation of organogenesis: Application to the effects of strain stimulus on collective cell behavior during ameloblast migration. J Theor Biol 262: 58-70.

48. Dernowsek JA, Rezende RA, Passamai VE, Noritomi PY, Kemmoku DT, et al. (2016) Tissue spheroids encaged into microscaffolds with internal structure to increase cell viability. Procedia CIRP 49: 174-177.

49. Yue XF, Wang L, Wang R (2013) Tissue modeling and analyzing with finite element method: A review for cranium brain imaging. Int $\mathrm{J}$ Biomed Imaging 2013: 1-12. 